\title{
Collagen types I and II distribution: a relevant indicator for the functional properties of articular cartilage in immobilised and remobilised rabbit knee joints
}

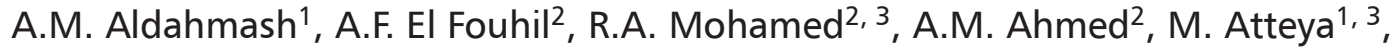 \\ S.A. Al Sharawy², R.A. Qureshi² \\ 'Stem Cell Unit, Department of Anatomy, College of Medicine, King Saud University, Riyadh, Saudi Arabia \\ ${ }^{2}$ Department of Anatomy, College of Medicine, King Saud University, Riyadh, Saudi Arabia \\ ${ }^{3}$ Department of Histology, Faculty of Medicine, Cairo University, Cairo, Egypt
}

[Received 12 June 2014; Accepted 17 September 2014]

\begin{abstract}
The objective of the present work was to study changes in collagen type I and type II distribution in the articular cartilage of immobilised and remobilised rabbit knee joints. Twenty-four adult male rabbits were divided into three groups. One of the groups was a control group with free movement. The right knee joints of animals of the other two groups were immobilised for 4 weeks, followed by a period of 10 weeks of remobilisation for animals of group 3. Collagen type I and type II in the articular cartilage of tibial medial condyle of the right knee joint were estimated immunohistochemically in all groups. A degenerative process was evident after 4 weeks of immobilisation of rabbit knee joint leading to a partial shift in the density of collagen composition from type II to type I. After a period of 10 weeks of remobilisation, regenerative processes, evidenced by a restoration of collagen type II to normal, proceeded on top of degenerative processes, evidenced by the significantly higher content of collagen type I compared with normal. The present study pointed to the importance of assessment of collagen distribution as a relevant indicator for the functional properties of articular cartilage in immobilised and remobilised joints. (Folia Morphol 2015; 74, 2: 169-175)
\end{abstract}

Key words: collagen type I, collagen type II, immobilised knee, immunohistochemistry

\section{INTRODUCTION}

The functional and structural integrity of cartilage tissue is believed to rely mainly on collagen type II molecules within its matrix [25]. Collagen type II in turn acts in concert with other collagens, types III, VI, IX, X, and XI [18], and matrix proteoglycans such as aggrecan [6].

Collagen type II protein has been localised in articular cartilage of many different species including man $[9,22]$. Collagen type II mRNA produced by chondrocytes has also been detected in vivo at the light microscopic level in mouse [26] and human cartilage $[1,16]$.

Some authors, for example, Gay et al. [9], Gebhard et al. [10], Mankin et al. [17], Miosge et al. [20], and Nerlich et al. [22], have demonstrated collagen type I or its corresponding mRNA in osteoarthritic cartilage.

With the help of immunohistochemistry, reverse-transcription-polymerase chain reaction, quantitative real-time-polymerase chain reaction, and in situ hybridisation, Miosge et al. [20] found that not only

Address for correspondence: Prof. A.F.I. El Fouhil, MD, MSc, PhD, Department of Anatomy and Embryology, College of Medicine, King Saud University, PO Box 2925 (28), Riyadh 11461, Saudi Arabia, tel: +966(11)4671314, mobile: +966501562983, fax: +966(11)4671300, 
collagen type II, but also collagen type I is synthesised by the cells of the diseased cartilage tissue, especially in the later stages of osteoarthritis.

Articular cartilage is composed of chondrocytes embedded within an extracellular matrix of collagens, proteoglycans, and non-collagenous proteins [19]. Loading and movement of the joint are of major importance for the maintenance of the morphologic and functional integrity of the articular cartilage [27]. Immobilisation of joint comprises a major component of treatment for various diseases whether for the purpose of stabilising fracture frequency or to rest inflamed or damaged tissues secondary to disease or traumatic injury $[2,4,28]$. The impact of immobilisation and unloading of joints on articular cartilage has been investigated in animal models $[2,11]$ and humans $[12,27,28]$. Previous studies have demonstrated that joint immobilisation and unloading lead to functional adaptation of articular cartilage that involve morphologic changes of chondrocytes and cartilage matrix $[13,15]$. Articular cartilage changes were detected as early as one week following immobilisation of rat knee joint [2, 3]. Studies performed on animals have also investigated the effect of remobilisation on the immobilisation-induced cartilage changes to detect the reversibility of these changes. The results of the studies were controversial. Following immobilisation of rabbit knees for 6 weeks, a period of 6 weeks of remobilisation was not sufficient to recover from the moderate alteration of proteoglycan produced [8]. Full restoration of articular cartilage proteoglycan concentration was not obtained even after 50 weeks of remobilisation in canine immobilised knee joints for 11 weeks [11]. Loss of chondrocytes was irreversible, while reduction of cartilage proteoglycan was almost reversible in immobilised rat knee joints for 4 weeks, after 16 weeks of remobilisation [3]. On the other hand, proteoglycan/collagen thickness was not found to change with 8 weeks of canine knee joint disuse followed by 3 weeks of remobilisation [15]. Chondrocytes provide $10 \%$ or less of the total volume of cartilage; consequently, the functional properties of cartilage, including stiffness, durability and distribution of load, rely on extracellular matrix, composed predominantly of collagen [14]. Collagen type II is the main collagen type of hyaline cartilage responsible for the stability and cell biological functions of healthy articular cartilage [21]. Collagen type I is normally synthesised by chondrocytes of the developing articular cartilageand gradually disappears in the postnatal period [5]. Such type of collagen was detected in degenerative joint diseases [20]. Previous studies have focused on chondrocytes and proteoglycan changes, but, to the best of the authors' knowledge, immobilisation-induced changes in collagen types and distribution, as well as, the effect of remobilisation on such changes were not studied immunohistochemically before. The aim of the present work was to conduct an immunohistochemical study to investigate the alteration in the distribution of collagen type I and collagen type II in the articular cartilage of the medial condyle of the tibia of the right knee joint of rabbits after a period of 4 weeks of immobilisation and to compare such distribution to that detected following a period of 10 weeks of remobilisation.

\section{MATERIALS AND METHODS}

The study was conducted in the Department of Anatomy, College of Medicine, King Saud University, Riyadh, Kingdom of Saudi Arabia. The study was approved by the Research Ethical Committee of the College of Medicine, King Saud University (approval No. 11/2870/IRB).

Animals. Twenty four adult male New Zeeland rabbits, aged about 7 weeks, were used in the present study. The body weight ranged $2.5-3.0 \mathrm{~kg}$. All rabbits underwent a 5 days acclimatisation period before the beginning of the experiment in the animal house of College of Medicine, King Saud University. The rabbits were kept in a room at constant temperature $\left(25 \pm 2^{\circ} \mathrm{C}\right)$ with light from 8:00 am to $8: 00 \mathrm{pm}$. All through the experiment, the rabbits were freely given tap water and commercially available foods. The study followed the International Guidelines for the Care and Use of Laboratory Animals for experimental procedure.

Study design. An experimental study was conducted on an animal model in which the right knee joints were immobilised followed by a period of remobilisation. The rabbits were allocated into three groups: Group 1 (12 animals): control group with free movement; 6 animals were sacrificed after 4 weeks (to be age-matched with animals of group 2) and the other 6 animals were sacrificed after 14 weeks (to be age-matched with animals of group 3); Group 2 (6 animals): immobilised group, in which the right knee joints were immobilised for 4 weeks, by applying a splint; Group 3 ( 6 animals): remobilised group, in which the right knee joints were immobilised for 4 weeks, by applying a splint, followed by a period of 10 weeks with remobilisation after removal of this splint.

Procedure. Immobilisation of knee joints, in extension position, was induced by applying a splint (Co-Flex ${ }_{N L}{ }^{-}$"Easy Tear; PATENTED TECHNOLOGY" 2 inches 
$x 5$ yds-Cat.\# 5200xx-036) on the hind-limb over the knee joint from the middle of the thigh to the middle of the hind-leg. The bandage allowed normal blood circulation to the hind-limb. Direct damage to the skin was prevented by the use of a soft pad of cotton that separated the splint from the skin of the animal. Rabbits were observed daily to ensure that the splints are in their positions without oedema of the hind-limbs [8].

Histological technique. At time of sacrifice, rabbits were anaesthetised by intramuscular injection of sodium pentobarbital ( $200 \mathrm{mg} / \mathrm{kg}$ body weight, Nembutal, Abbott Laboratories, North Chicago, IL) and then decapitated. From each animal, the right knee joint was resected. The proximal epiphysis of the tibia was dissected and sawed sagittally at the level of the medial condyle. The specimens of the bone of tibia were fixed in $10 \%$ neutral buffered formalin in $0.1 \mathrm{M}$ phosphate buffer ( $\mathrm{pH} \mathrm{7.4)}$ for $24 \mathrm{~h}$. Specimens were then decalcified in $5 \%$ formic acid in $10 \%$ neutral buffered formalin for 7 days. The formic acid decalcification solution was changed daily. The specimens were processed and embedded in paraffin blocks to prepare $5-\mu \mathrm{m}$-thick paraffin sections. After deparaffinisation in xylene and hydration in descending grades of ethanol, these sections were stained with haematoxylin and eosin (H\&E) for routine histological examination.

Immunohistochemical technique for detection of type I and type II collagen. Paraffin sections were deparaffinised, rehydrated and washed in $0.05 \mathrm{M}$ tris-buffered saline (TBS) pH 7.6. Immunostaining was performed using a mouse monoclonal collagen type I antibody (Clone No. COL-1, ab 6308, Abcam) and a mouse monoclonal collagen type II antibody (Clone No. II-II6B3-s and cll cl-c, Developmental Hybridoma Bank, University of lowa, IA, USA) at dilutions of 1:500 and 1:50, respectively, for $1 \mathrm{~h}$ at room temperature. Negative controls were performed by omission of the primary antibody. Sections were then incubated with NovoLink Polymer Detection System (product No. RE 7280-K; Leica Biosystems, Newcastle, United Kingdom) for 30 min to detect any tissue-bound primary antibody. Sections were further incubated with the substrate/chromogen, 3,3-diaminobenzidine (DAB), prepared from Novocastra DAB chromogen $(50 \mu \mathrm{L})$ and $1 \mathrm{~mL}$ of NovoLink Substrate Buffer (Polymer). Washing with TBS (0.05 M, pH 7.6) twice for 5 min was performed following each step of incubation. Immunostained sections were then counterstained with Mayer's haematoxylin. Finally, sections were dehydrated rapidly in ascending grades of ethanol, cleared in xylene and mounted in distyrene plasticizer xylene mounting medium. The stained sections were evaluated and photographed by means of an Olympus BX51 bright field microscope equipped with an Olympus DP72 camera (Olympus Corporation, Japan).

Image analysis. High-resolution whole-slide digital scans of all collagen type I and collagen type II immunostained sections were created with a ScanScope scanner (Aperio Technologies, Inc.). The digital slide images were then viewed and analysed using the viewing and image analysis tools of Aperio's ImageScope software. Five areas, each with the fixed size of $137,000 \mu \mathrm{m}^{2}$, were randomly selected per section. To quantify the immunopositivity, the colour deconvolution (colour separation) algorithm (Aperio Technologies, Inc.) was set up (by colour calibration) so as to detect and quantify only the brown colour of DAB positive staining. The algorithm was then run on the selected area to measure the percentage of immumopositivity relative to the area being measured. The analysis output results were finally exported to Excel sheets and subjected to statistical analysis.

\section{Statistical analysis}

Data collected were subjected to statistical analysis using SPSS (PASW Statistics 18) software where analysis of variance (ANOVA) was used for an overall comparison between the study groups and the post hoc Tukey HSD test was used for pair wise comparisons. Differences were considered significant when $p$ was equal to or less than 0.05 . A $95 \%$ confidence level was used to calculate a confidence interval, which is a range of values around the mean where the "true" (population) mean can be expected to be located, with $95 \%$ certainty.

\section{RESULTS}

Histological results. H\&E stained sections of tibial articular cartilage of the right knee joint from control animals (Group 1) showed normal architecture of chondrocytes inside lacunae and extracellular cartilage matrix, with no evidence of degeneration (Fig. 1A, B). After 4 weeks of immobilisation, the proximal part of the tibial articular cartilage of immobilised group (Group 2) showed a thick zone of intense acidophilic staining of cartilage matrix and numerous young flattened chondrocytes lying with their longitudinal axes parallel to the surface of the articular cartilage. The distal part of the articular cartilage, however, still has 

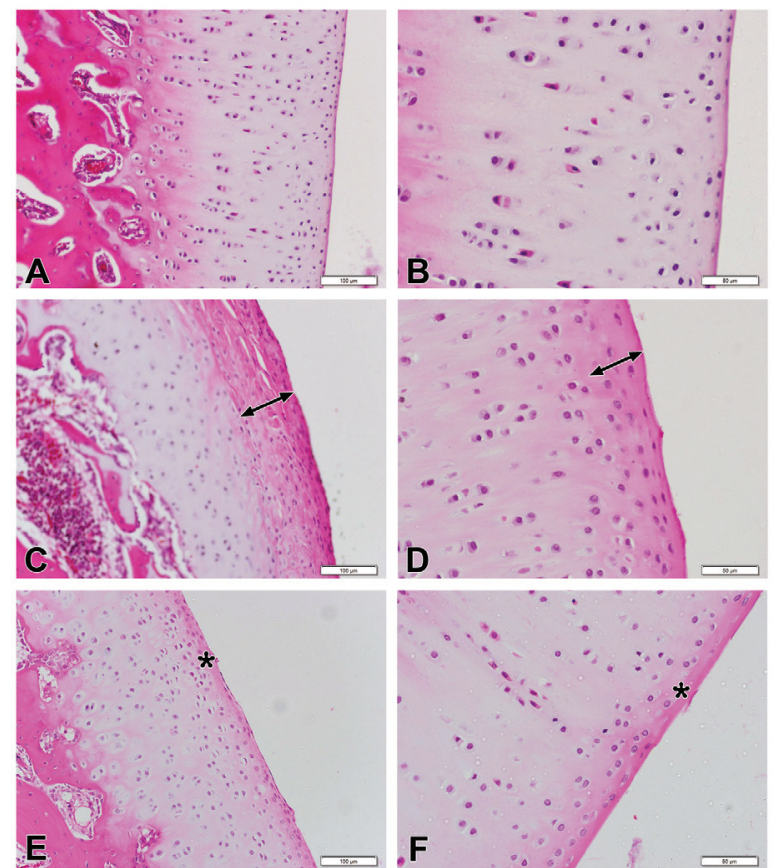

Figure 1. Photomicrographs of the proximal epiphysis of tibia stained with H\&E; A. Low magnification of tibial articular cartilage of a control rabbit, showing chondrocytes separated by basophilic cartilage matrix in the proximal part of the section (right side of the photograph). The distal part of the section (left side of the photograph) shows cancellous bone; scale bar $=100 \mu \mathrm{m}$; B. High magnification of the previous photomicrograph, showing the proximal part of the articular cartilage with chondrocytes inside lacunae and basophilic cartilage matrix; scale bar $=50 \mu \mathrm{m} ;$ C. Low magnification of tibial articular cartilage of an immobilised rabbit, showing a thick zone of intense acidophilia of cartilage matrix in the proximal part of the articular cartilage (double-headed arrow). The distal part of the articular cartilage, however, still has a basophilic cartilage matrix; scale bar $=100 \mu \mathrm{m}$; D. High magnification of the previous photomicrograph, showing the proximal part of the articular cartilage with a thick zone of intense acidophilia of cartilage matrix (double-headed arrow) and numerous young flattened chondrocytes lying with their longitudinal axes parallel to the surface of the articular cartilage. The distal part of the articular cartilage, however, still has a basophilic cartilage matrix and mature chondrocytes arranged in columns perpendicular to the surface of the articular cartilage; scale bar $=50 \mu \mathrm{m} ; \mathrm{E}$. Low magnification of tibial articular cartilage of a remobilised rabbit, showing a thin zone of less intense acidophilia of cartilage matrix in the proximal part of the articular cartilage (asterisk; scale bar $=100 \mu \mathrm{m}$; F. High magnification of the previous photomicrograph, showing the proximal part of the articular cartilage with a thin zone of less intense acidophilia of cartilage matrix (asterisk) and fewer young flattened chondrocytes lying with their longitudinal axes parallel to the surface of the articular cartilage; scale bar $=50 \mu \mathrm{m}$.

a basophilic cartilage matrix and mature chondrocytes arranged in columns perpendicular to the surface of the articular cartilage (Fig. 1C, D). After additional 10 weeks of free remobilisation, the proximal part of the tibial articular cartilage of remobilised group (Group 3) showed reduction of the acidophilic staining of cartilage matrix and fewer young flattened chondrocytes lying with their longitudinal axes parallel to the surface of the articular cartilage (Fig. 1E, F).
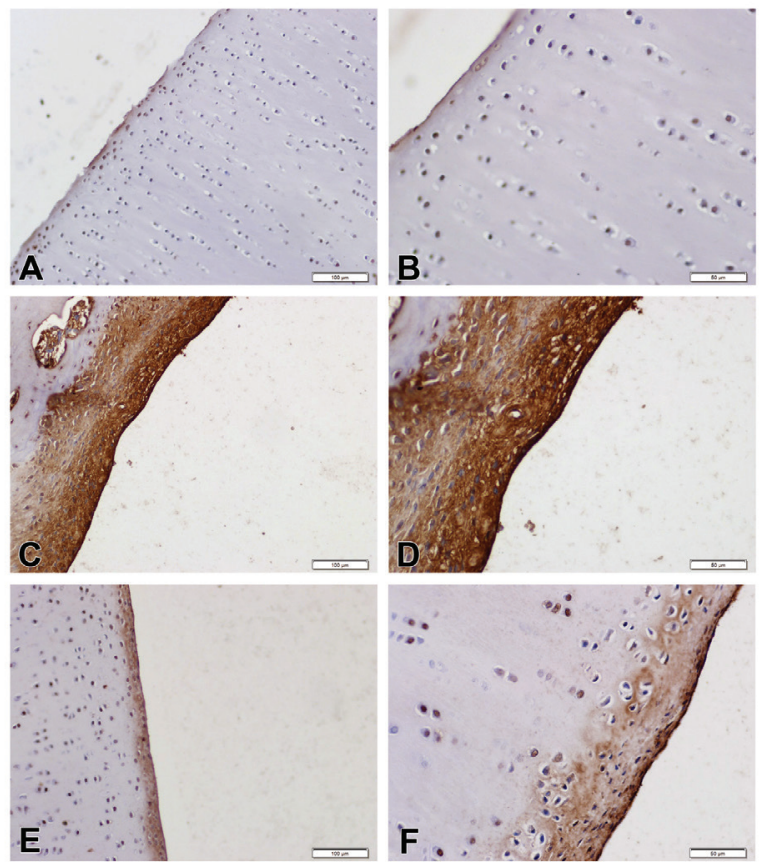

Figure 2. Photomicrographs of the proximal epiphysis of tibia immunostained with anti-collagen I; A, B. Low and high magnifications, respectively, of tibial articular cartilage of a control rabbit, showing scarce immunoreactivity in the most proximal part; C, D. Low and high magnifications, respectively, of tibial articular cartilage of an immobilised rabbit, showing a thick zone of intense immunoreactivity in the proximal part of the articular cartilage;

E, F. Low and high magnifications, respectively, of tibial articular cartilage of a remobilised rabbit, showing marked reduction of immunoreactivity in the proximal part compared with the immobilised group; scale bars $=100 \mu \mathrm{m}$ for $\mathrm{A}, \mathrm{C}, \mathrm{E}$ and $50 \mu \mathrm{m}$ for $\mathrm{B}, \mathrm{D}, \mathrm{F}$.

Immunohistochemical results. The positive immunostaining for collagen type I in tibial articular cartilage of knee joint of control animals was sparse and its intensity was very weak throughout the cartilage matrix (Fig. 2A, B). After 4 weeks of immobilisation, collagen type I expression was increased markedly throughout most of the tibial articular cartilage (Fig. 2C, D). After 10 weeks of remobilisation, collagen type I expression in the articular cartilage showed marked reduction compared with the immobilised group (Fig. 2E, F), but remained higher than that of the control group. Immunostaining for collagen type II in tibial articular cartilage of knee joint of control animals was positive throughout the cartilage matrix. This immunoreactivity was intensely positive in the territorial areas especially around the cell clusters and moderate in the inter-territorial areas (Fig. 3A, B). Afterimmobilisation for 4weeks, collagen typell immunoreactivity showed marked reduction in the inter-territorial matrix, while the territorial areas remained intensely positive (Fig. 3C, D). After free remobilisation for 10 weeks, collagen type II expression increased in 


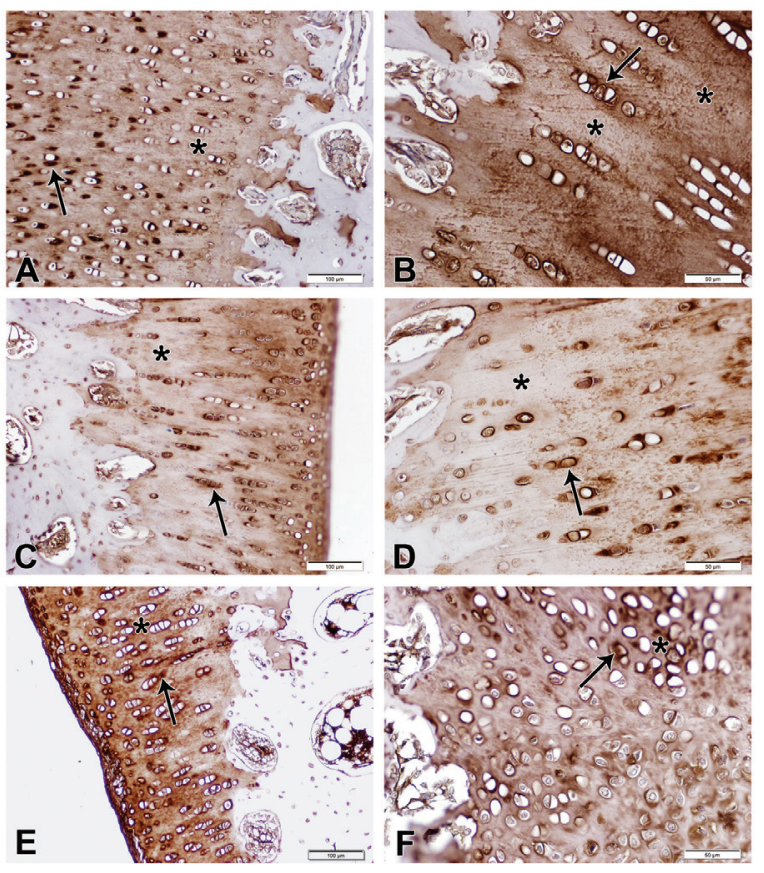

Figure 3. Photomicrographs of the proximal epiphysis of tibia immunostained with anti-collagen II; A, B. Low and high magnifications, respectively, of tibial articular cartilage of a control rabbit, showing intense immunoreactivity in the territorial (arrows) and less intense immunoreactivity in the inter-territorial (asterisks) areas; C, D. Low and high magnifications, respectively, of tibial articular cartilage of an immobilised rabbit, showing marked reduction of immunoreactivity in territorial (arrows) and inter-territorial (asterisks) areas, in comparison with the control group; E, F. Low and high magnifications, respectively, of tibial articular cartilage of a remobilised rabbit, showing an increase of immunoreactivity in territorial (arrows) and inter-territorial (asterisks) areas, in comparison with the immobilised group; scale bars $=100 \mu \mathrm{m}$ for $\mathrm{A}, \mathrm{C}, \mathrm{E}$ and $50 \mu \mathrm{m}$ for $\mathrm{B}, \mathrm{D}, \mathrm{F}$.

the inter-territorial matrix. Its intensity was comparable to control animals (Fig. 3E, F).

Image analysis and statistical results. The area per cent of collagen type I immunopositivity showed a highly statistically significant increase in immobilised group compared with the control group $(p=0.0004)$. In the remobilised group, the area per cent of collagen type I immunopositivity was markedly reduced compared with the immobilised group and the difference was statistically significant ( $p=0.0093$ ), but it remained significantly higher compared with the control group $(p=0.009)$ (Table 1). The area per cent of collagen type II immunopositivity was significantly reduced in the immobilised compared with the control group $(p=0.0126)$. The area per cent of collagen type II immunopositivity went up in the remobilised group, with a statistically significant difference compared with the immobilised group $(p=0.0191)$, and reached the control level (Table 1).

\section{DISCUSSION}

In the present study, twenty-four 7-week-old male New Zeeland white rabbits were used. The rabbit is a well-accepted model for histological, biochemical and biomechanical analyses of in vitro and in vivo cartilage and bone repair [5]. Male rabbits were used, in the present study, because in females collagen type II is possibly influenced by oestrogen level, where oestrogen counters the acceleration of type II collagen degradation [23]. At the age of 7 weeks, the essential adult pattern of articular cartilage was established in rabbits in which only traces of type I collagen could be detected [5]. The present study was designed to immobilise the rabbit knee joints for 4 weeks, a period shorter than that reported in the literature, and to remobilise them for 10 weeks, a period longer than that reported in previous studies performed on rabbit knees. The aim was to detect early changes in type and distribution of collagen and to study the possibility of complete reversibility of such changes after a relatively longer period of remobilisation.

The present study showed positive immunostaining for collagen type II throughout the extracellular matrix

Table 1. Mean area per cent of collagen I and II immunopositivity in articular cartilage of the studied groups

\begin{tabular}{|c|c|c|c|c|c|c|}
\hline \multirow[t]{2}{*}{ Percent positive } & \multicolumn{2}{|c|}{ Group 1} & \multicolumn{2}{|c|}{ Group 2} & \multicolumn{2}{|c|}{ Group 3} \\
\hline & Mean \pm SD & $95 \% \mathrm{Cl}$ & Mean \pm SD & $95 \% \mathrm{Cl}$ & Mean \pm SD & $95 \% \mathrm{Cl}$ \\
\hline Collagen I & $4.150 \pm 1.477$ & $2.316-5.984$ & $26.090 \pm 7.772$ & $16.440-35.740$ & $15.679 \pm 3.706$ & $11.077-20.281$ \\
\hline P1 & & & $0.0002^{*}$ & & $0.009 *$ & \\
\hline P2 & $0.0002^{*}$ & & & & $0.017^{*}$ & \\
\hline Collagen II & $99.657 \pm 0.161$ & $99.457-99.857$ & $98.022-1.127$ & $96.623-99.421$ & $99.776 \pm 0.049$ & $99.715-99.837$ \\
\hline P1 & & & $0.0126^{*}$ & & 0.9725 & \\
\hline P2 & $0.0126^{*}$ & & & & $0.0191 *$ & \\
\hline
\end{tabular}

${ }^{*}$ Significant difference $(\mathrm{p} \leq 0.05)$; SD — standard deviation; $\mathrm{Cl}$ - confidence interval; $\mathrm{P} 1$ - vs. group 1; P2 — vs. group 2 
with intense immunoreactivity in the territorial area of tibial articular cartilage of knee joints of the control group. On the other hand, sparse areas of positive immunostaining for type I collagen were detected throughout the articular cartilage. Previous studies, performed in animals [21] and in humans [7, 24], reported that all cartilage matrices are composed predominantly of collagen type II.

H\&E sections of animals of immobilised group showed that the intensity of acidophilic staining of the matrix in the superficial layer of tibial articular cartilage increased compared with animals of the control group, coincident with a significant increase in collagen type I expression in immunostained sections of the same group compared with controls. On the other hand, immunoreactive collagen type II expression was significantly decreased in the interterritorial matrix of tibial articular cartilage of immobilised group compared with the control group. $H \& E$ sections of the tibial articular cartilage of right knee joint of the remobilised group showed reduction of the intensity of acidophilic staining of cartilage matrix in the superficial layer compared to that of the immobilised group, but still more intensely stained than that of the control group. Immunostained sections showed that collagen type I decreased significantly in the remobilised compared with the immobilised group, but remained significantly increased compared with control group. Furthermore, collagen type II increased significantly in remobilised group compared with immobilised group and returned to control level. The present study suggested a partial shift of collagen synthesis by chondrocytes from type II to type I as a result of immobilisation of the knee joint. Previous studies reported increasing amount of collagen type I in osteoarthritis and suggested it as one of the factors involved in the pathogenesis of such disease [20]. Immobilisation of rabbit's knee might provoke a degenerative process similar to osteoarthritis [8]. Degenerative processes were evident after 4 weeks of immobilisation. Remobilisation of the knee joint for 10 weeks causes an increase in the amount of collagen type II toward normal, indicating regenerative efforts on the part of the chondrocytes to compensate for the collagen type II loss. Furthermore, collagen type I decreased in amount, but was still significantly higher than normal, indicating that degenerative process was still proceeding. It seems that the reversibility of immobilisation-induced changes in collagen distribution depends on the duration of immobilisation itself and not on the duration of remobilisation. Once degenerative process begins, the normal pattern of collagen in articular cartilage will never be restored. To the best of the authors' knowledge, the present study is the first immunohistochemical one describing the alteration in the distribution of collagen type I and collagen type II in the articular cartilage following immobilisation and remobilisation. Furthermore, the present study pointed to the irrelevance of previous animal studies reporting the reversibility of immobilisation-induced articular cartilage changes $[3,14]$. The conclusion of such studies was based on the proteoglycan content which should not be considered as a relevant indicator for the functional properties of the articular cartilage.

Regarding the limitations of the study, the authors did not study collagen distribution following an immobilisation period shorter than 4 weeks to assess more accurately the time of beginning of the degeneration processes.

\section{CONCLUSIONS}

In conclusion, a degenerative process was evident as early as 4 weeks following immobilisation of rabbit knee joint leading to a partial shift in the density of collagen composition from type II to type I. After a period of 10 weeks of remobilisation, regenerative processes, evidenced by a restoration of collagen type II to normal, proceeded on top of the ongoing degenerative processes, evidenced by the significantly higher content of collagen type I compared with normal. A much longer remobilisation period seemed not to be of great benefit regarding complete reversibility of such case. Further studies on collagen distribution following shorter periods of immobilisation to indicate the approximate period after which degenerative process proceeds, and hence, safe interval during which remobilisation should start, would be recommended.

The present study pointed to the importance of assessment of collagen distribution as a relevant indicator for the functional properties of articular cartilage in immobilised and remobilised joints. Identifying the causes and progress of the articular cartilage changes would give clinicians an insight into the onset and duration of rehabilitation of immobilised joint. Finally, clinicians should be aware that immobilisation of knee joints would cause irreversible changes in the collagen of the articular cartilage, and that remobilisation, as soon as possible, would be recommended.

\section{ACKNOWLEDGEMENTS}

The Hybridoma or monoclonal antibody developed by (Investigator: Thomas F. Linsenmayer) was obtained from the developmental Studies Hybridoma Bank, developed under the auspices of the NICHD and 
maintained by the university of lowa, Department of Biology, lowa City, IA 52242.

\section{REFERENCES}

1. Aigner T, Vornehm SI, Zeiler G, Dudhia K, von der Mark K, Bayliss MT (1997) Suppression of cartilage matrix gene expression in upper zone chondrocytes of osteoarthritic cartilage. Arthritis Rheum, 40: 562-569.

2. Ando A, Suda H, Hagiwara Y, Onoda Y, Chimoto E, Itoi E (2012) Remobilization does not restore immobilization-induced adhesion of capsule and restricted joint motion in rat knee joints. Tohoku J Exp Med, 227: 13-22.

3. Ando A, Suda H, Hagiwara Y, Onoda Y, Chimoto E, Saijo $Y$, Itoi E (2011) Reversibility of immobilization-induced articular cartilage degeneration after remobilization in rat knee joints. Tohoku J Exp Med, 224: 77-85.

4. Brandt KD (2003) Response of joint structures to inactivity and to reloading after immobilization. Arthritis Rheum, 49: 267-271

5. Buma $P$, Pieper JS, van Tienen $T$, van Susante $\mathrm{JL}$, van der Kraan PM, Veerkamp JH van den Berg WB, Veth RP, van Kuppevelt TH (2003) Cross-linked type I and type II collagenous matrices for the repair of full-thickness articular cartilage defects-a study in rabbits. Biomaterials, 24: 3255-3263.

6. Doege KJ, Sasaki M, Kimura T, Yamada Y (1991) Complete coding sequence and deduced primary structure of the human cartilage large aggregating proteoglycan, aggrecan. Human- specific repeats, and additional alternatively spliced forms. J Biol Chem, 266: 894-902.

7. Eyre D (2002) Collagen of articular cartilage. Arthritis Res, 4: 30-35.

8. Fu LL, Maffulli N, Chan KM (2001) Intra-articular hyaluronic acid following knee immobilisation for 6 weeks in rabbits. Clin Rheumatol, 20: 98-103.

9. Gay S, Müller P, Lemmen C, Remberger K, Matzen K, Kühn K (1986) Immunohistochemical study on collagen in cartilage-bone metamorphosis and degenerative osteoarthritis. Klin Wochenschr, 54: 969-976.

10. Gebhard PM, Gehrsitz A, Bau B, Soder S, Eger W, Aigner T (2003) Quantification of expression levels of cellular differentiation markers does not support a general shift in the cellular phenotype of osteoarthritic chondrocytes. J Orthop Res, 21: 96-101.

11. Haapala J, Arokoski JP, Rönkkö S, Agren U, Kosma VM, Lohmander LS, Tammi M, Helminen HJ, Kiviranta I (2001) Decline after immobilisation and recovery after remobilisation of synovial fluid IL1, TIMP, and chondroitin sulphate levels in young beagle dogs. Ann Rheum Dis, 60: 55-60.

12. Hudelmaier $M$, Glaser $C$, Hausschild A, Burgkart R, Eckstein $F(2006)$ Effects of joint unloading and reloading on human cartilage morphology and function, muscle cross-sectional areas, and bone density: a quantitative case report. J Musculoskelet Neuronal Interact, 6: 284-290.

13. Iqbal K (2012) Effects of immobilization on chondrocytes and pericellular matrix in articular cartilage of patella in rats. J Morphol Sci, 29: 8-11.

14. Jadin KD, Wong BL, Bae WC, Li KW, Williamson AK, Schumacher BL, Price JH, Sah RL (2005) Depth-varying density and organization of chondrocytes in immature and mature bovine articular cartilage assessed by $3 d$ imaging and analysis. J Histochem Cytochem, 53: 1109-1119.

15. LeRoux MA, Cheung HS, Bau JL, Wang JY, Howell DS, Setton LA (2001) Altered mechanics and histomorphometry of canine tibial cartilage following joint immobilization. Osteoarthr Cartil, 9: 633-640.

16. Lui VC, Ng U, Nicholls J, Tam PP, Cheah KS (1995) Tissue-specific and differential expression of alternatively spliced alpha 1(II) collagen mRNAs in early human embryos. Dev Dyn, 203: 198-211.

17. Mankin HJ, Dorfman H, Lippiello L, Zarins A (1971) Biochemical and metabolic abnormalities in articular cartilage from osteoarthritic human hips. II. Correlation of morphology with bio- chemical data. J Bone Joint Surg Am, 53: 523-537.

18. Mayne R (1989) Cartilage collagens. Arthritis Rheum, 32: 241-246.

19. Methoefer K, Williams RJ, Warren RF, Wickiewicz TL, Marx R (2006) High-impact athletics after knee articular cartilage repair: a prospective evaluation of the microfracture technique. Am J Sports Med, 34: 1413-1418.

20. Miosge N, Hartmann M, Maelicke C, Herken R (2004) Expression of collagen type I and type II in consecutive stages of human osteoarthritis. Histochem Cell Biol, 122: 229-236.

21. Naumann A, DennisJE, Awadallah A, Carrino DA, Mansour JM, Kastenbauer E, Caplan Al (2002) Immunochemical and mechanical characterization of cartilage subtypes in rabbit. J Histochem Cytochem, 50: 1049-1058.

22. Nerlich AG, Wiest I, von der Mark K (1993) Immunohistochemical analysis of interstitial collagens in cartilage of different stages of osteoarthrosis. Virchows Archiv B Cell Pathol, 63: 249-255.

23. Oestergaard $S$, Sondergaard BC, Hoegh-Andersen $P$, Henriksen K, Qvist P, Christiansen C, Tankó LB, Karsdal MA (2006) Effects of ovariectomy and estrogen therapy on type II collagen degradation and structural integrity of articular cartilage in rats: implications of the time of initiation. Arthritis Rheum, 54: 2441-2451.

24. Pearle AD, Warren RF, Rodeo SA (2005) Basic science of articular cartilage and osteoarthritis. Clin Sports Med, 24: 1-12.

25. Prockop DJ, Kivirikko KI, Tuderman L, Guzman NA (1979) The biosynthesis of collagen and its disorders. N Engl J Med, 301: 13-23.

26. Sandell $\sqcup$, Nalin AM, Reife RA (1994) Alternative splice form of type II procollagen mRNA (IIA) is predominant in skeletal precursors and non-cartilaginous tissues during early mouse development. Dev Dyn, 199: 129-140.

27. Vanwanseele B, Eckstein F, Knecht H, Stüssi E, Spaepen A (2002) Knee cartilage of spinal cord-injured patients displays progressive thinning in the absence of normal joint loading and movement. Arthritis Rheum, 46: 2073-2078.

28. Vanwanseele B, Lucchinetti E, Stüssi E (2002) The effects of immobilization on the characteristics of articular cartilage: current concepts and future directions. Osteoarthr Cartil, 10: 408-419. 